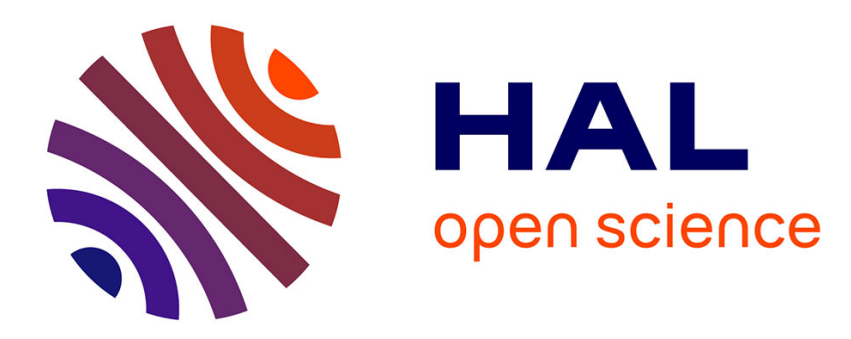

\title{
Periodic and quasiperiodic vortex shedding in the wake of a rotating sphere
}

\author{
Benoît Pier
}

\section{To cite this version:}

Benoît Pier. Periodic and quasiperiodic vortex shedding in the wake of a rotating sphere. Journal of Fluids and Structures, 2013, 41, pp.43-50. 10.1016/j.jfluidstructs.2012.09.002 . hal-00733514

\section{HAL Id: hal-00733514 https://hal.science/hal-00733514}

Submitted on 19 Sep 2012

HAL is a multi-disciplinary open access archive for the deposit and dissemination of scientific research documents, whether they are published or not. The documents may come from teaching and research institutions in France or abroad, or from public or private research centers.
L'archive ouverte pluridisciplinaire HAL, est destinée au dépôt et à la diffusion de documents scientifiques de niveau recherche, publiés ou non, émanant des établissements d'enseignement et de recherche français ou étrangers, des laboratoires publics ou privés. 


\title{
Periodic and quasiperiodic vortex shedding in the wake of a rotating sphere
}

\author{
Benoît PIER* \\ Laboratoire de mécanique des fluides et d'acoustique \\ École centrale de Lyon - CNRS - Université Claude-Bernard Lyon 1 - INSA Lyon \\ 36 avenue Guy-de-Collongue, 69134 Écully cedex, France
}

\begin{abstract}
The flow past a sphere rotating about an axis aligned with the streamwise direction is numerically investigated. The dynamics is governed by the incompressible Navier-Stokes equations and depends on two control parameters: the Reynolds number Re and rotation rate $\Omega$. The present investigation systematically covers the range $\operatorname{Re} \leq 350$ and $\Omega \leq 2$. First, the axisymmetric steady base flow (whether stable or not) is computed for all values of the control parameters. Then, after linearization of the equations about the base flow, the growth rates and frequencies of the leading eigenmodes are obtained. Fully nonlinear direct numerical simulations yield the detailed flow fields and hydrodynamic forces acting on the sphere. Different wake modes (low-frequency periodic helical, quasiperiodic shedding and high-frequency periodic helical) are identified and their characteristic frequencies precisely determined.
\end{abstract}

Keywords: wake, vortex shedding, drag, lift, quasiperiodic, bifurcation, instability

\section{Introduction}

At moderate Reynolds numbers, our understanding of the wake dynamics for a fixed sphere in uniform upstream flow is by now fairly complete. More complex scenarios prevail when additional effects are taken into account, such as shear in the oncoming flow, the presence of a wall, rotation of the obstacle or nonspherical shapes. Most of these configurations break the axisymmetry of the formulation. The purpose of the present investigation is to shed new light on the dynamics prevailing in a situation governed by two control parameters but preserving the axisymmetry of the problem: the wake of a sphere rotating about an axis aligned with the incident flow.

The bifurcation scenario followed by the wake of a fixed sphere in uniform upstream flow is now fairly well established, both experimentally and numerically (Nakamura, 1976; Sakamoto and Haniu, 1995; Johnson and Patel, 1999; Ghidersa and Duček, 2000; Schouveiler and Provansal, 2002; Thompson et al., 2001): at low Reynolds numbers a steady, axisymmetric flow prevails; beyond a first critical Reynolds number, $\operatorname{Re}_{1} \simeq 212$, the flow bifurcates and a steady non-axisymmetric wake with planar symmetry is selected; beyond a second critical Reynolds number, $\mathrm{Re}_{2} \simeq 272$, periodic shedding sets in, but conserves the symmetry plane. At still larger Reynolds numbers, the planar symmetry is broken (Mittal, 1999), and the wake becomes progressively disordered and turbulent (Ormières and Provansal, 1999; Tomboulides and Orszag, 2000; Constantinescu and Squires, 2004). Careful measurements of the hydrodynamic forces (drag, lift, torque) acting on the sphere allow characterization of these different flow régimes (Maxworthy, 1965; Benjamin, 1993; Bouchet et al., 2006).

In many situations of practical interest, the incoming flow is not perfectly uniform. In the presence of shear (Dandy and Dwyer, 1990; Kurose and Komori, 1999; Kim et al., 2005; Kim, 2006; Bagchi and Balachandar, 2002a), strain (Bagchi and Balachandar, 2002b) or stratification (Hanazaki, 1988), the lack of

*E-mail: benoit.pier@ec-lyon.fr 
axisymmetry modifies the bifurcation scenario and the hydrodynamic forces. If the obstacle is not fixed but allowed to interact with the flow, it may rotate and rise or fall under the action of torque and gravity (Bagchi and Balachandar, 2002a; Jenny et al., 2003; Jenny and Dušek, 2004; Jenny et al., 2004; Veldhuis et al., 2005; Fernandes et al., 2007; Ern et al., 2012). Numerous studies have also addressed the wake of deformable bodies such as bubbles or droplets (Legendre and Magnaudet, 1997; Kurose et al., 2001; Magnaudet et al., 2003; Legendre et al., 2006; Sugioka and Komori, 2007; Rastello et al., 2009, 2011).

Of particular interest in the present context are the flows around axisymmetric but non-spherical bodies. When the symmetry axis of disks or ellipsoids is aligned with the incident flow, the problem remains axisymmetric and the wake dynamics depend not only on the Reynolds number but also on the aspect ratio. For the extreme case of an infinitely thin disk, Fabre et al. (2008) have identified new vortex shedding modes and introduced a symmetry-based model to explain this scenario and predict the evolution of the lift force. For a thicker disk, yet more régimes have been found (Auguste et al., 2010). Meliga et al. (2009) use the leading eigenmodes derived from global stability theory and develop a weakly nonlinear model that accurately predicts the sequence of bifurcations for a thin disk. The efficiency of this model relies, among other things, on the fact that the leading eigenmodes have very similar growth rates, favouring (weak) nonlinear interactions which control the complex bifurcation scenario. Inspired by these findings, the present investigation revisits the configuration used by Kim and Choi (2002): the wake of a sphere rotating about a streamwise oriented axis. The rotation of the sphere introduces a chirality in the problem but does not break the axisymmetry. The growth rates of the leading eigenmodes depend on two parameters, Reynolds number and rotation rate, and competition between these is expected to lead to rich dynamics, possibly amenable to weakly nonlinear interaction models.

The paper is organized as follows. After formulating the problem and presenting the numerical methods in $\S 2$, axisymmetric base flows and their linear stability properties are discussed in $\S 3$. The different finiteamplitude vortex shedding régimes and associated hydrodynamic forces are presented in $\S 4$. Finally, $\S 5$ summarizes the results.

\section{Problem formulation and numerical method}

The study is carried out using the incompressible Navier-Stokes equations. The Reynolds number is defined as $\operatorname{Re}=U_{\infty} D / \nu$, where $U_{\infty}$ is the free-stream velocity, $D$ the sphere diameter and $\nu$ the kinematic viscosity.

Throughout this investigation, cylindrical coordinates are used with $r, \theta$ and $z(u, v$ and $w)$ denoting radial, azimuthal and axial coordinates (velocities) respectively. The $z$-axis is aligned with the free-stream velocity and the origin is at the center of the sphere. For later use, a Cartesian $(x, y, z)$-frame is also defined. Using non-dimensional variables based on $U_{\infty}$ and $D$, the total velocity and pressure fields are denoted by $\mathbf{u}(r, \theta, z, t)$ and $p(r, \theta, z, t)$ respectively and are governed by the momentum and continuity equations,

$$
\begin{aligned}
\partial_{t} \mathbf{u}+(\mathbf{u} \cdot \nabla) \mathbf{u}+\nabla p & =\frac{1}{\operatorname{Re}} \Delta \mathbf{u}+\mathbf{f}, \\
\nabla \cdot \mathbf{u} & =0
\end{aligned}
$$

with boundary conditions

$$
\begin{array}{rll}
u=v-\Omega r=w=0 & \text { for } & r^{2}+z^{2}=1 / 4 \\
u=v=w-1=0 & \text { for } & r \rightarrow \infty \quad \text { or } \quad z \rightarrow \pm \infty .
\end{array}
$$

Here $\Omega$ is the non-dimensional rotation rate (based on $U_{\infty}$ and $D$ ) of the sphere about the $z$-axis. The dynamics of the rotating-sphere wake are then completely determined by two control parameters, Re and $\Omega$.

The numerical method closely follows the technique successfully implemented for studying the nonrotating sphere wake (Pier, 2008). An immersed boundary method (Fadlun et al., 2000; Mittal and Iaccarino, 2005; Zhang and Zheng, 2007) is used, whereby the presence of the sphere is enforced through the externally applied volume force $\mathbf{f}$ in the momentum equation (1). Thus, the entire space is assumed to be filled 


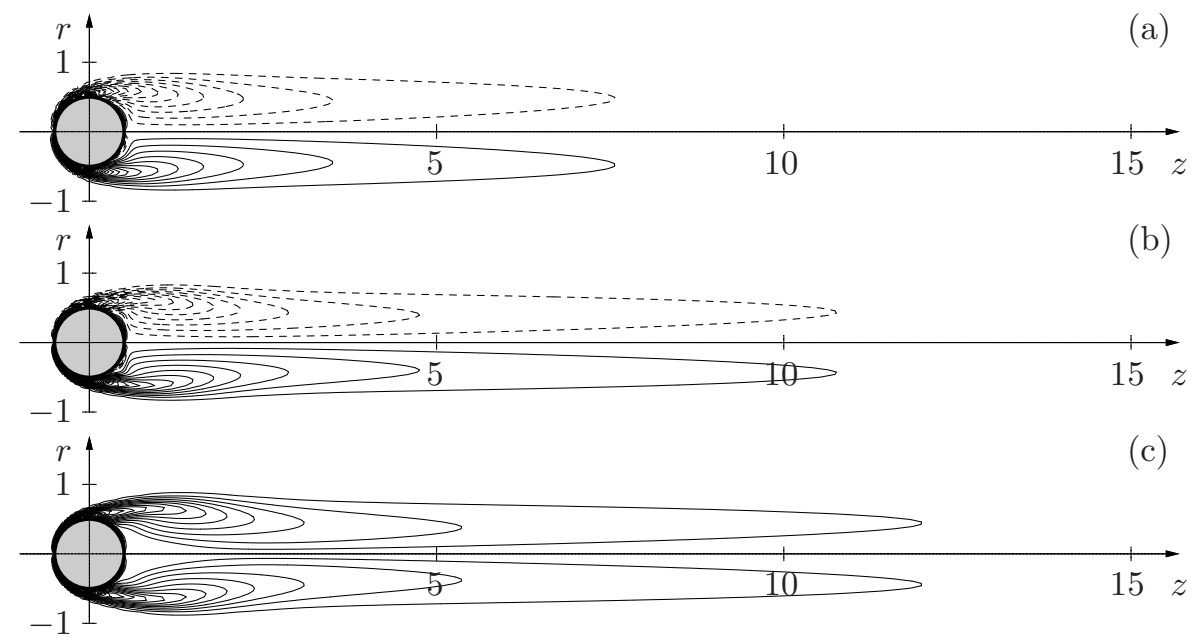

Figure 1: Flow structure of the basic axisymmetric wake for (a) $\operatorname{Re}=150$ and $\Omega=1$, (b) $\operatorname{Re}=250$ and $\Omega=1$, (c) $\operatorname{Re}=250$ and $\Omega=2$. Solid (dashed) isolines correspond to positive (negative) values of azimuthal vorticity, spaced by 0.5 .
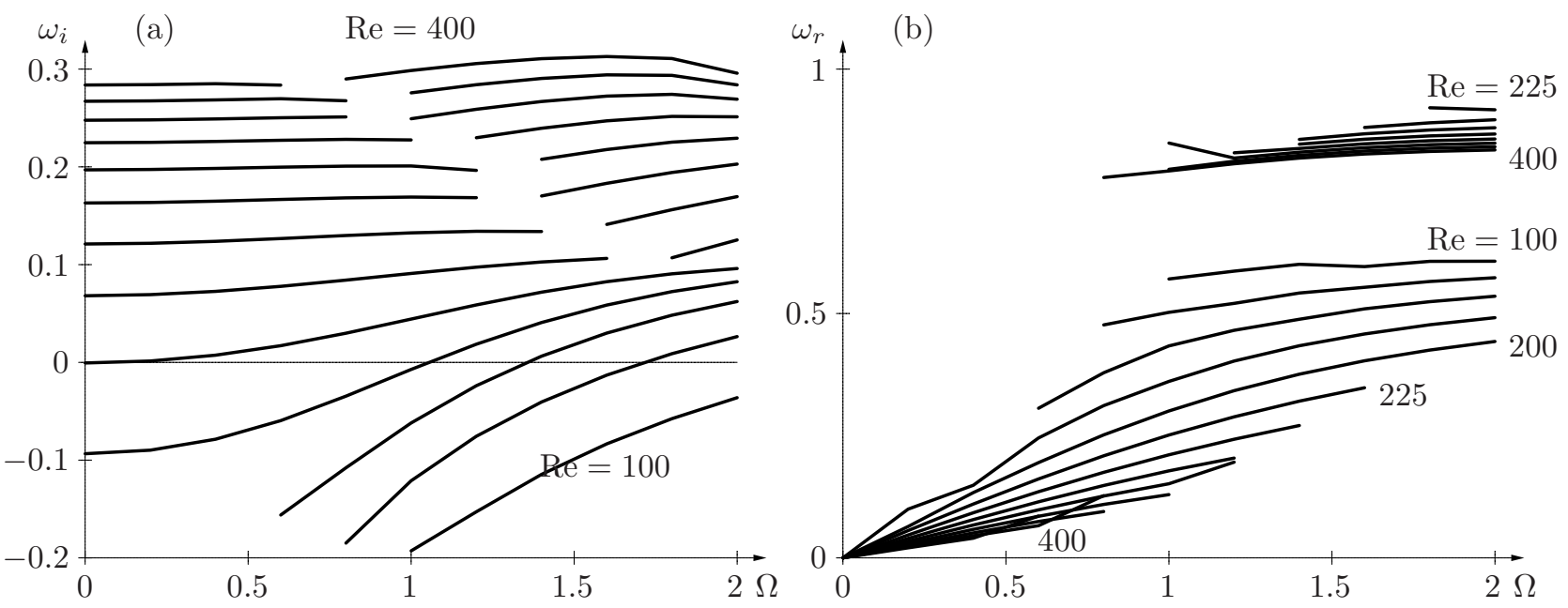

Figure 2: (a) Growth rate $\omega_{i}$ and (b) frequency $\omega_{r}$ of the leading eigenmode for axisymmetric basic wakes, computed for $\Omega=0.0,0.2, \ldots, 2.0$ and $\operatorname{Re}=100,125, \ldots, 400$.

with fluid and the body force ensures that the boundary conditions (3) of a rotating sphere are met. All flow fields are Fourier-expanded in the azimuthal coordinate $\theta$, while the $(r, z)$-plane is discretized on a Cartesian grid using finite-differences in $z$ and Chebyshev collocation points in $r$. The time-marching algorithm uses a second-order accurate predictor-corrector fractional-step method, similar to (Hugues and Randriamampianina, 1998).

\section{Axisymmetric base flows and linear stability}

Axisymmetric wakes have been computed by retaining only the axisymmetric component in the azimuthal Fourier expansions. For all Reynolds numbers and rotation rates considered in the present study, the sphere wakes were found to approach a steady state when time-marching the governing equations $(1,2)$.

The structure of the basic axisymmetric wake for different values of the control parameters is illustrated in figure 1 by isolines of the azimuthal vorticity $\omega_{\theta}=\partial_{z} u-\partial_{r} w$.

The linear stability of these axisymmetric wakes is probed by computing the response to a non-axisymmetric perturbation. Here only a single non-axisymmetric azimuthal Fourier component is retained in the 

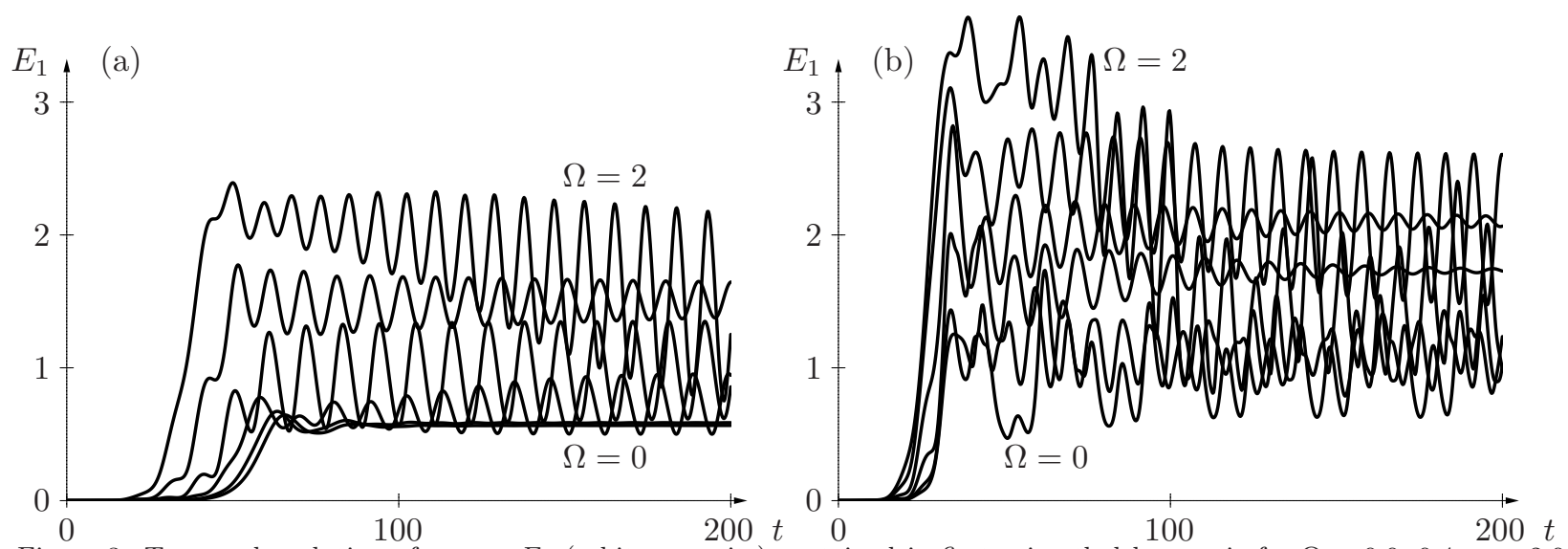

Figure 3: Temporal evolution of energy $E_{1}$ (arbitrary units) contained in first azimuthal harmonic for $\Omega=0.0,0.4, \ldots, 2.0$ and $\operatorname{Re}=250$ (a), $\operatorname{Re}=325$ (b).

expansions of the flow fields, and the Navier-Stokes equations are linearized around the previously computed basic flow. Growth rates and frequencies of the most unstable modes are then derived from the time-series of selected flow components, recorded at a fixed spatial location. Such a flow component $f$ is expected to evolve as $f \propto \exp (-\mathrm{i} \omega t)$, where $\omega$ is the complex eigenvalue associated with the mode. The growth rate $\omega_{i}$ is then obtained by a linear fit of $\log |f|$, while the frequency $\omega_{r}$ is obtained by spectral analysis of the compensated $f \exp \left(-\omega_{i} t\right)$. Thus, the growth rates $\omega_{i}$ and frequencies $\omega_{r}$ are obtained for the most unstable mode at each setting of the control parameters Re and $\Omega$. These values are shown in figure 2 . It is observed that two distinct mode types lead to instability, depending on the control parameters: at moderate rotation rates and low Reynolds numbers, the instability is dominated by a "slow" mode, the frequency of which scales nearly linearly with the sphere rotation rate $\Omega$. In contrast, at higher parameter values, a "fast" mode dominates, whose frequency is approximately independent of $\Omega$. Similar behaviour is observed for the nonlinear dynamics, as discussed below.

\section{Nonlinear dynamics}

To investigate the nonlinear dynamics, a finite number of azimuthal Fourier harmonics are retained and the direct numerical simulations take into account the nonlinear coupling between all these modes. When starting integration, the initial condition is chosen as the previously computed axisymmetric base flow with a small non-axisymmetric perturbation. In situations where this axisymmetric flow is unstable, the nonaxisymmetric perturbation starts to grow exponentially in time. After a transient growth phase, nonlinear effects come into play that limit the amplitude growth. At large times, the system is found to approach a periodic or quasiperiodic régime, or to display irregular behaviour.

Monitoring the temporal evolution of the energy $E_{1}$ contained in the first azimuthal harmonic illustrates the development of non-axisymmetric components in the sphere wake. In Figure 3 , the energy content $E_{1}$ is plotted for $0 \leq \Omega \leq 2$ and $\operatorname{Re}=250$ and 325 . For the wakes corresponding to these plots, after entering a finite-amplitude régime, the energy $E_{1}$ is seen to reach either a constant value or to converge towards a state of periodic oscillations. At larger values of the Reynolds number, irregular oscillations may also be found to persist indefinitely.

To further characterize the flow dynamics, the hydrodynamic forces acting on the sphere have been computed. These forces are obtained by spatial integration of the volume force used in the immersed boundary method; there is no need to evaluate components of the stress tensor at the sphere surface. The drag coefficient $C_{z}$ measures, in non-dimensional units, the component of the force acting in the $z$-direction aligned with the outer flow. The lift coefficients $C_{x}$ and $C_{y}$ are obtained by projection onto the $x$ - and $y$-axes respectively, while the lateral force coefficient $C_{l}$ is defined as $C_{l}=\sqrt{C_{x}^{2}+C_{y}^{2}}$. 


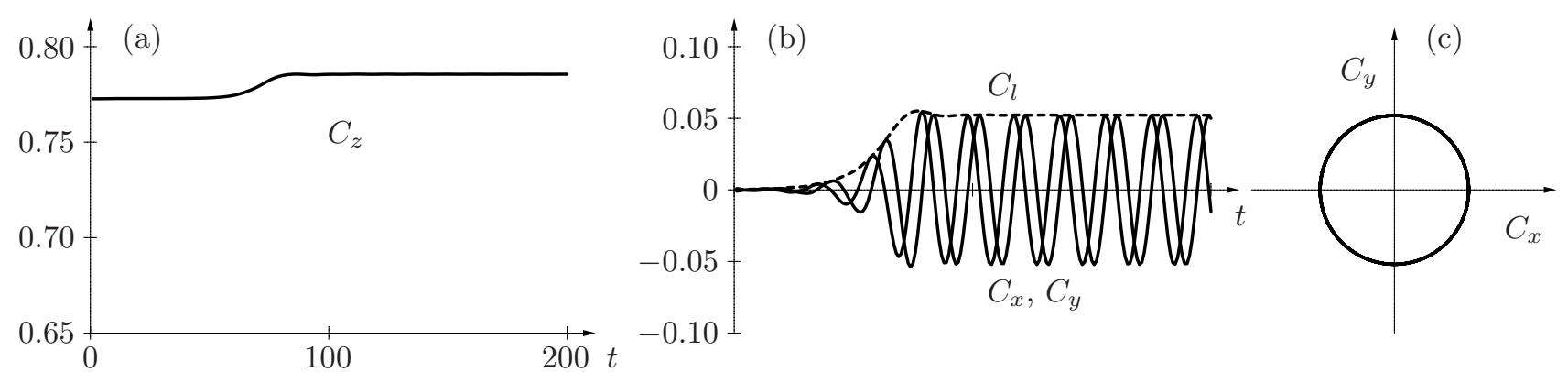

Figure 4: Temporal evolution of hydrodynamic forces for Re $=225$ and $\Omega=1$. $C_{z}: \operatorname{drag} ; C_{x}$ and $C_{y}$ : lift forces; $C_{l}$ : transverse force. Initial condition consists up of the axisymmetric base flow with a small-amplitude non-axisymmetric perturbation.

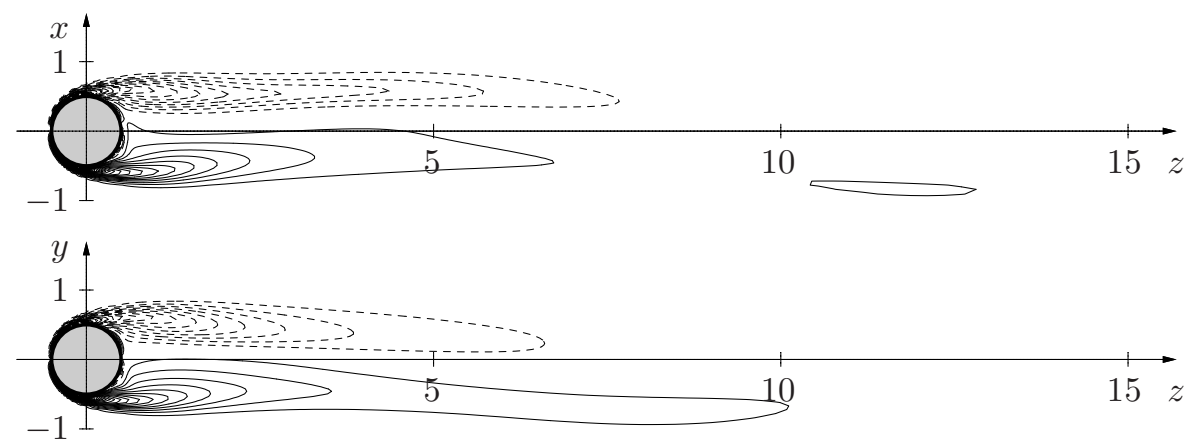

Figure 5: Snapshot of vorticity fields in the helical régime at $R e=225$ and $\Omega=1$. Isolines of azimuthal vorticity in two orthogonal planes.

For axisymmetric wakes, all coefficients vanish except the $\operatorname{drag} C_{z}$. In configurations where the axisymmetric base flow is unstable, the development of finite non-axisymmetric flow components is accompanied by a similar development of transverse forces, characterized by $C_{x}$ and $C_{y}$ (and $C_{l}$ ). The constant, periodic, quasiperiodic or irregular values taken by these hydrodynamic force coefficients characterize the associated wake dynamics.

\subsection{Helical régime}

The wake behaviour observed for $\operatorname{Re}=225$ and $\Omega=1$ is typical of the dynamics prevailing after the first destabilization of the axisymmetric flow. Figure 4 illustrates the temporal evolution of the force coefficients, starting from the slightly perturbed (and unstable) axisymmetric base flow. After a transient régime characterized by growth of transverse force components, the wake is seen to approach a state of constant drag, slightly higher than for the base flow (figure $4 \mathrm{a}$ ). Lift coefficients $C_{x}$ and $C_{y}$ display harmonic oscillations, out of phase by a quarter-period, while the magnitude of the lateral force $C_{l}$ is observed to tend to a constant value (figure $4 \mathrm{~b})$. This is further illustrated by the time-trace in the $\left(C_{x}, C_{y}\right)$-plane (figure $4 \mathrm{c}$ ): beyond the transient phase, a perfect circle is described at a constant angular speed.

The spatial structure of the wake flow is illustrated in figure 5, where isolines of the azimuthal vorticity are plotted for two orthogonal $(x, z)$ - and $(y, z)$-planes.

Temporal spectral analysis of the force coefficients (as well as of any other flow components) demonstrates that this régime is characterized by a single frequency. For $\operatorname{Re}=225$ and $\Omega=1$, the periodicity of the lift coefficients is obtained as $\omega_{x}=\omega_{y}=0.31$. In fact, it can be shown that the entire wake is in a helical state, characterized by "solid-body" rotation of the flow field about the $z$-axis at constant angular speed. This means that the flow is steady in a frame of reference rotating about the $z$-axis at $\omega_{x}\left(=\omega_{y}\right)$. Note that the angular speed $\omega_{x}=\omega_{y}$ is well below the sphere rotation rate $\Omega=1$.

\subsection{Quasi-periodic vortex shedding}

For $\operatorname{Re}=275$ and $\Omega=0.8$, a different behaviour is obtained. Again, the development of non-axisymmetric components is accompanied by an increase in drag. But here, no steady state is reached: the drag coefficient 

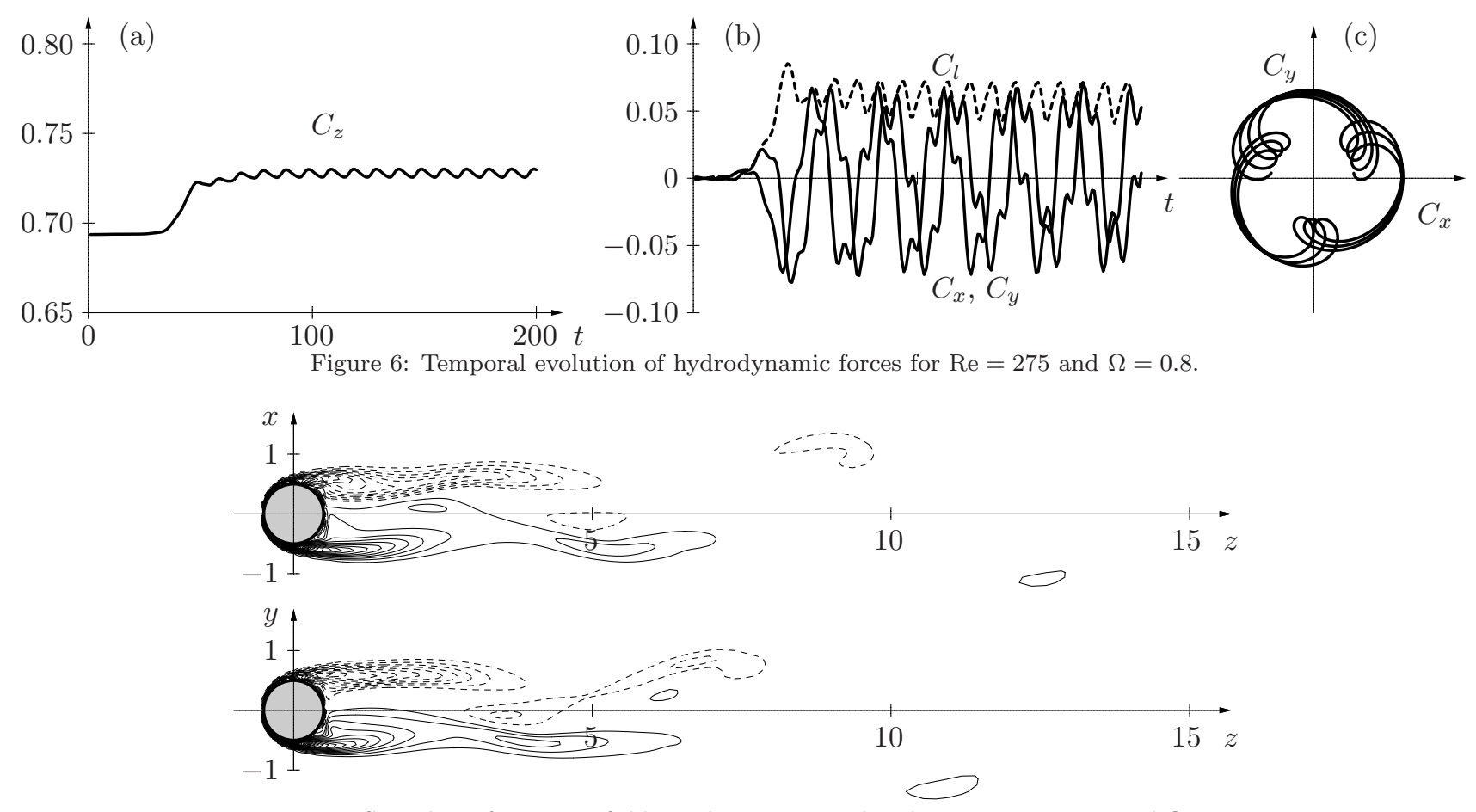

Figure 7: Snapshot of vorticity fields in the quasiperiodic régime at $\operatorname{Re}=275$ and $\Omega=0.8$.

continues to oscillate (figure 6a). Lift coefficients $C_{x}$ and $C_{y}$ display quasiperiodic oscillations while the lateral force $C_{l}$ fluctuates with the same periodicity as the drag (figure $6 \mathrm{~b}$ ). This behaviour leads to a more complex pattern in the $\left(C_{x}, C_{y}\right)$-plane, see figure $6(\mathrm{c})$. Temporal spectral analyses show that these signals are characterized by two distinct (and incommensurate) frequencies: $\omega_{x}=\omega_{y}=0.21$ and $\omega_{z}=\omega_{l}=0.62$. Indeed, $C_{z}$ and $C_{l}$ are periodic (with same frequency $\omega_{z}=\omega_{l}$ ) while $C_{x}$ and $C_{y}$ are quasiperiodic (displaying a combination of $\omega_{z}$ and $\left.\omega_{x}\right)$.

A snapshot of the spatial structure of the associated vorticity fields is given in figure 7 . This dynamics can be interpreted as a quasiperiodic vortex shedding régime, corresponding to the combination of a helical mode ("solid-body rotation" about the $z$-axis at $\omega_{x}$ ) and vortex shedding waves travelling axially downstream (frequency $\omega_{z}$ ).

\subsection{High-frequency helical régime}

For $\operatorname{Re}=300$ and $\Omega=1$ a further wake behaviour is observed, representative of a third class of flow dynamics. Afer a relatively long transient, the system approaches a (single-frequency) periodic state. The $\operatorname{drag} C_{z}$ and the lateral force $C_{l}$ reach constant values, while the lift coefficients $C_{x}$ and $C_{y}$ display harmonic oscillations in quadrature, leading to a circular time-trace in the $\left(C_{x}, C_{y}\right)$-plane (figure 8). This régime is again of periodic helical vortex shedding type, characterized by a single frequency $\omega_{x}=\omega_{y}=0.90$. Note that the frequency of this "solid-body" rotation is quite closer to the sphere rotation rate $\Omega$. Hence, this régime could be termed "high-frequency helical vortex shedding".

The corresponding vorticity fields are illustrated for two orthogonal planes in figure 9. Although this régime is periodic and the flow fields would be steady in a frame rotating at $\omega_{x}$ around the $z$-axis, these vorticity fields closely resemble those prevailing in the quasiperiodic régime (see figure 7) and are rather different from those of the low-frequency helical régime (see figure 5). It is as if the axial vortex shedding and the helical "solid-body" rotation were locked together, or "frozen" (Kim and Choi, 2002).

\subsection{Characteristic frequencies}

For each Reynolds number and sphere rotation rate, the characteristic frequencies have been determined via temporal Fourier analyses of long time series of the force coefficients. The helical frequencies $\omega_{x}\left(=\omega_{y}\right)$ 

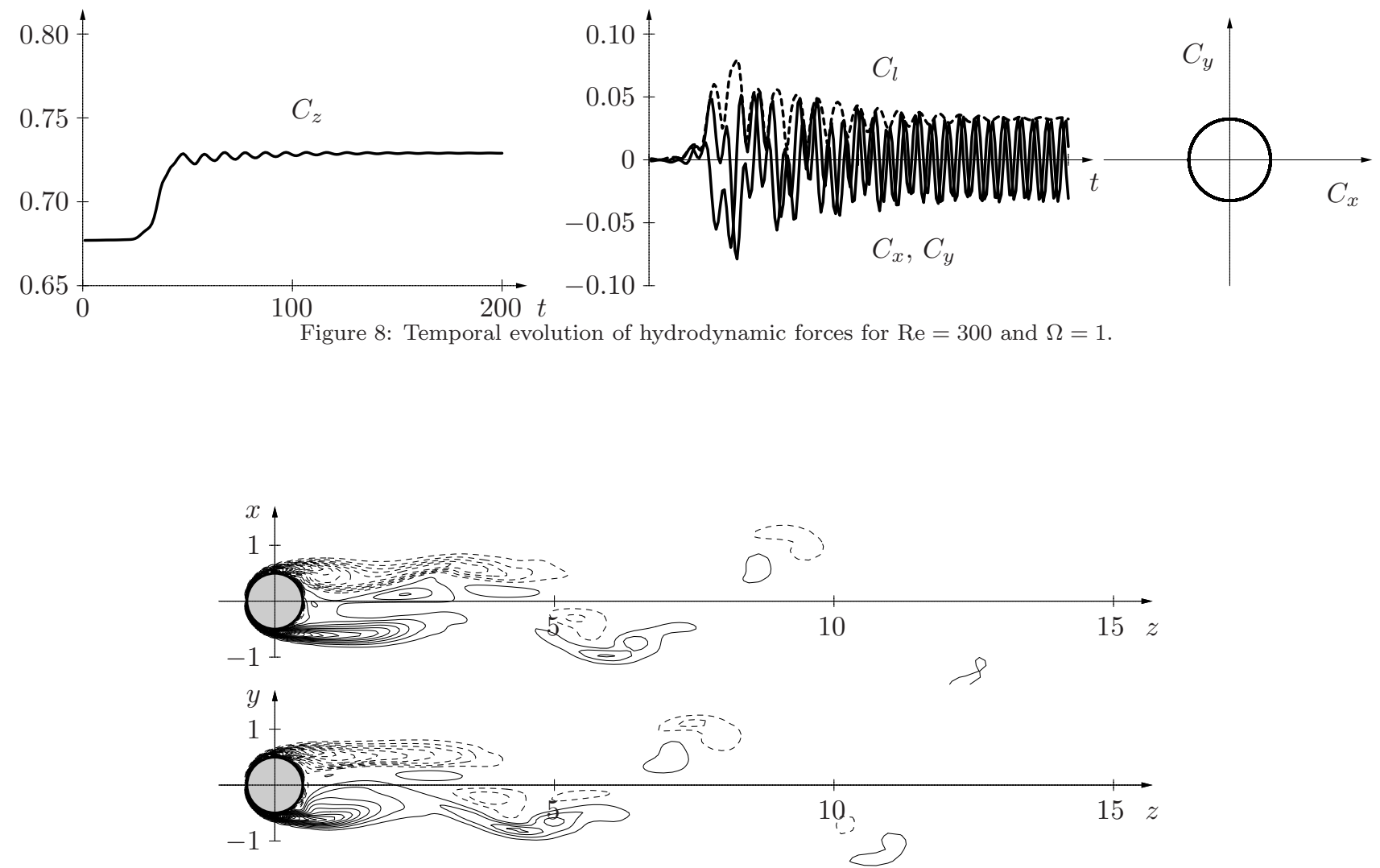

Figure 9: Snapshot of vorticity fields in the high-frequency helical régime at $\operatorname{Re}=300$ and $\Omega=1$.
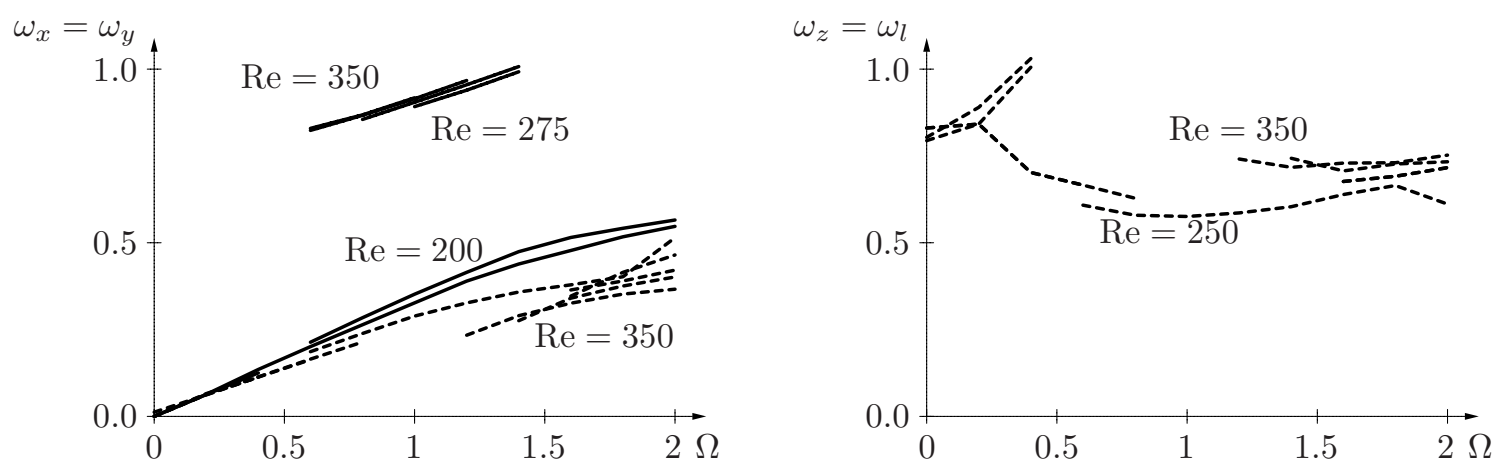

Figure 10: Characteristic frequencies prevailing in the rotating-sphere wake. (a) Frequencies $\omega_{x}=\omega_{y}$ dominating the fluctuations of the lift coefficients $C_{x}$ and $C_{y}$. (b) Frequencies $\omega_{z}=\omega_{l}$ governing the oscillations of the drag and lateral force coefficients $C_{z}$ and $C_{l}$. Solid curves correspond to low- or high-frequency helical régimes. Dashed curves indicate quasiperiodic vortex-shedding (or disordered) régimes. 


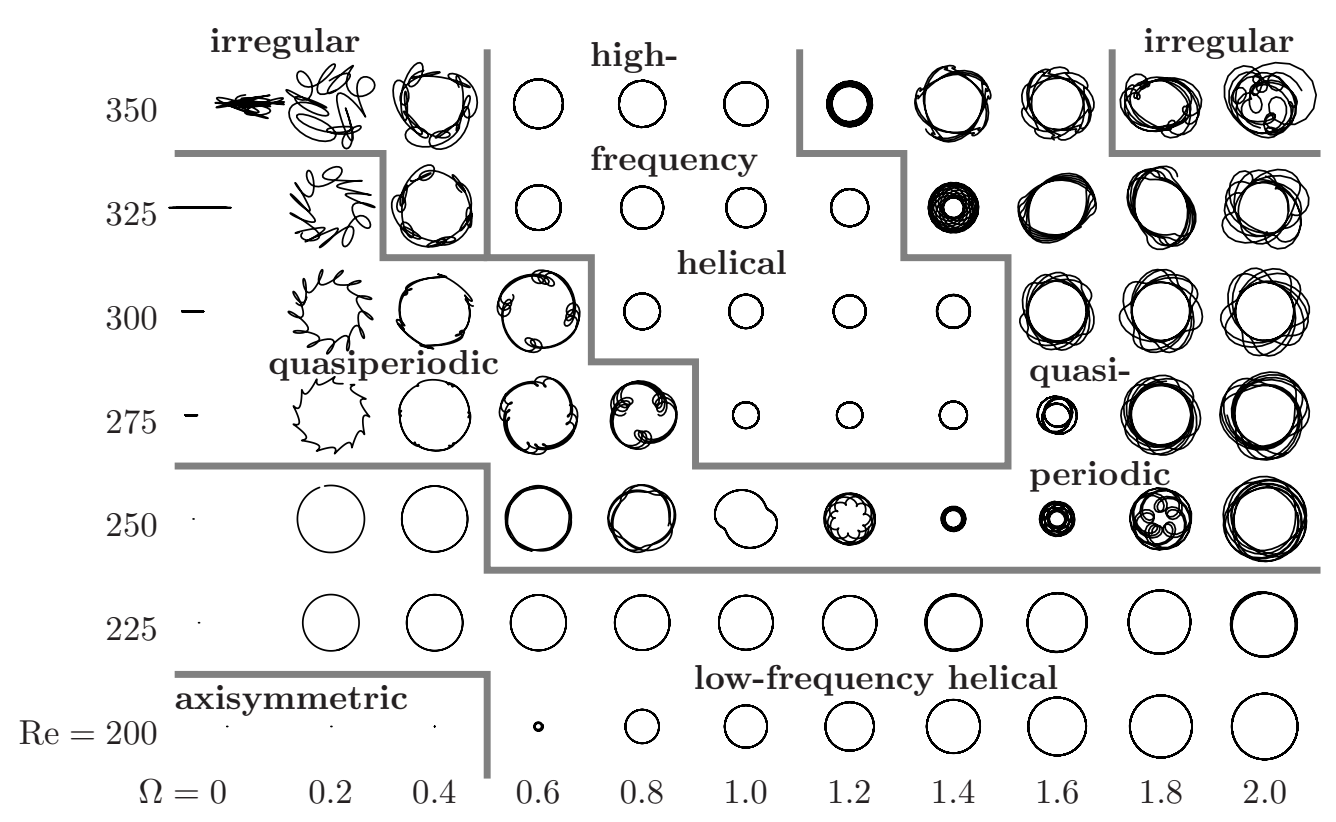

Figure 11: Map of the different régimes as a function of the control parameters.

are plotted in figure 10(a), while the axial frequencies $\omega_{z}\left(=\omega_{l}\right)$ are shown in figure 10(b). In these plots, solid curves correspond to low- and high-frequency modes while dashed curves indicate quasiperiodic (or disordered) vortex shedding. Note that helical frequencies dominating the fluctuations of the lift coefficients $C_{x}$ and $C_{y}$ are obtained for any non-axisymmetric flow, while the axial vortex-shedding frequencies governing the oscillations of the drag and lateral force coefficients $C_{z}$ and $C_{l}$ are only relevent in the quasiperiodic shedding régimes.

In figure 10(a), it is seen that $\omega_{x}$ displays an almost linear dependence on $\Omega$ in the low-frequency helical and quasiperiodic régimes. Transition from low-frequency helical to quasiperiodic vortex shedding hardly affects these values. In the high-frequency helical régimes, however, order of magnitude larger values for $\omega_{x}$ are obtained. The axial frequencies $\omega_{z}$ shown in 10(b), correspond more specifically to axially travelling vortex shedding waves and display only weak dependence on the rotation rate $\Omega$.

\section{Conclusion and discussion}

Direct numerical simulations have been carried out in order to systematically cover the governing parameter space for sphere rotation rates $\Omega \leq 2$ and Reynolds numbers up to $\operatorname{Re}=350$. Figure 11 presents a map of the observed régimes characterized by the associated time-traces of the lift coefficients in the $\left(C_{x}, C_{y}\right)$-plane.

At low Reynolds numbers, the axisymmetric wake is stable. When the Reynolds number is increased, a low-frequency helical régime takes over, characterized by constant values of drag $\left(C_{z}\right)$ and transverse force $\left(C_{l}\right)$. The flow field is found to rotate around the $z$-axis at constant frequency $\omega_{x}=\omega_{y}$ without deformation. Indeed, in such a rotating frame, the flow field would be time-independent. The rate $\omega_{x}$ at which the wake rotates around the axis is found to increase almost linearly with the sphere rotation rate $\Omega$, and this régime can be viewed as a continuous deformation, through axial rotation, of the well-documented steady planar symmetric state for non-rotating spheres in the range $\operatorname{Re}_{1}<\mathrm{Re}<\mathrm{Re}_{2}$, with $\mathrm{Re}_{1} \simeq 212$ and $\mathrm{Re}_{2} \simeq 272$ (Johnson and Patel, 1999; Mittal, 1999; Ghidersa and Duček, 2000; Schouveiler and Provansal, 2002).

A second bifurcation occurs when the Reynolds number is increased, leading to a quasiperiodic state which can be interpreted as a modulation (at a second incommensurate frequency $\omega_{z}$ ) of the previous helical régime. A rotating frame in which the flow field would be steady no longer exists. Again, this régime can be viewed as the continuation through axial rotation of the periodic vortex shedding régime that prevails 
for $R e>R_{2} \simeq 272$ for a non-rotating sphere. In the non-rotating case, onset of vortex shedding occurs through a Hopf bifurcation (Schouveiler and Provansal, 2002). Here, our results indicate that this remains true along the entire boundary separating the low-frequency helical wakes from the quasiperiodic wakes. However, many more computations would be necessary to prove that the amplitude of the second-frequency component scales as the square-root of the distance to this critical boundary.

The third type of behaviour, termed the high-frequency helical régime, occurs at still larger Reynolds numbers. This periodic régime does not have an analogue in the non-rotating $\Omega=0$ case. While the transition from the low-frequency helical to the quasiperiodic régime is a continuous process, the switching from quasiperiodic to high-frequency helical régimes is discontinuous in the control parameters. Indeed, the dominant $\omega_{x}$-frequency prevailing in the wake abruptly increases while the amplitude of the transverse forces $\left(C_{l}\right)$ suddenly drops. The nature of the associated bifurcation remains unclear. Despite several attempts at slowly modifying one of the control parameters, no hysteresis was found.

At yet larger Reynolds numbers, irregular states have been observed. No systematic survey of the parameter space beyond $\mathrm{Re}=350$ has been attempted since this would require much finer spatial meshes to obtain reliable results.

In future work, it would be interesting to address the nature of the bifurcations between the different régimes in more detail and to test whether the theory of Meliga et al. (2009) can be adapted to the present configuration.

\section{Acknowledgements}

Computing resources from Fédération lyonnaise de calcul hautes performances (FLCHP) and Institut national de physique nucléaire et de physique des particules (IN2P3) are gratefully acknowledged.

\section{References}

Auguste, F., Fabre, D., Magnaudet, J., 2010. Bifurcations in the wake of a thick circular disk. Theoretical and Computational Fluid Dynamics 24, 305-313.

Bagchi, P., Balachandar, S., 2002a. Effect of free rotation on the motion of a solid sphere in linear shear flow at moderate Re. Physics of Fluids 14, 2719-2737.

Bagchi, P., Balachandar, S., 2002b. Steady planar straining flow past a rigid sphere at moderate Reynolds number. Journal of Fluid Mechanics 466, 365-407.

Benjamin, T. B., 1993. Note on formulas for the drag of a sphere. Journal of Fluid Mechanics 246, $335-342$.

Bouchet, G., Mebarek, M., Dušek, J., 2006. Hydrodynamic forces acting on a rigid fixed sphere in early transitional regimes. European Journal of Mechanics - B/Fluids 25, 321-336.

Constantinescu, G., Squires, K., 2004. Numerical investigations of flow over a sphere in the subcritical and supercritical regimes. Physics of Fluids 16, 1449-1466.

Dandy, D. S., Dwyer, H. A., 1990. A sphere in shear flow at finite Reynolds number: effect of shear on particle lift, drag, and heat transfer. Journal of Fluid Mechanics 216, 381-410.

Ern, P., Risso, F., Fabre, D., Magnaudet, J., 2012. Wake-induced oscillatory paths of bodies freely rising or falling in fluids. Annual Review of Fluid Mechanics 44, 97-121.

Fabre, D., Auguste, F., Magnaudet, J., 2008. Bifurcations and symmetry breaking in the wake of axisymmetric bodies. Physics of Fluids, 051702 .

Fadlun, E. A., Verzicco, R., Orlandi, P., Mohd-Yusof, J., 2000. Combined immersed-boundary finite-difference methods for three-dimensional complex flow simulations. Journal of Computational Physics 161, 35-60.

Fernandes, P. C., Risso, F., Ern, P., Magnaudet, J., 2007. Oscillatory motion and wake instability of freely rising axisymmetric bodies. Journal of Fluid Mechanics 573, 479-502.

Ghidersa, B., Duček, J., 2000. Breaking of axisymmetry and onset of unsteadiness in the wake of a sphere. Journal of Fluid Mechanics 423, 33-69.

Hanazaki, H., 1988. A numerical study of three-dimensional stratified flow past a sphere. Journal of Fluid Mechanics 192, 393-419.

Hugues, S., Randriamampianina, A., 1998. An improved projection scheme applied to pseudospectral methods for the incompressible Navier-Stokes equations. International Journal of Numerical Methods in Fluids 28, 501-521.

Jenny, M., Bouchet, G., Dušek, J., 2003. Nonvertical ascension or fall of a free sphere in a Newtonian fluid. Physics of Fluids 15, L9-L12.

Jenny, M., Dušek, J., 2004. Efficient numerical method for the direct numerical simulation of the flow past a single light moving spherical body in transitional regimes. Journal of Computational Physics 194, 215-232. 
Jenny, M., Dušek, J., Bouchet, G., 2004. Instabilities and transition of a sphere falling or ascending freely in a Newtonian fluid. Journal of Fluid Mechanics 508, 201-239.

Johnson, T. A., Patel, V. C., 1999. Flow past a sphere up to a Reynolds number of 300. Journal of Fluid Mechanics 378, 19-70.

Kim, D., Choi, H., 2002. Laminar flow past a sphere rotating in the streamwise direction. Journal of Fluid Mechanics 461, $365-386$.

Kim, D., Choi, H., Choi, H., 2005. Characteristics of laminar flow past a sphere in uniform shear. Physics of Fluids 17 , 103602.

Kim, I., 2006. Forces on a spherical particle in shear flow at intermediate Reynolds numbers. International Journal of Computational Fluid Dynamics 20, 137-141.

Kurose, R., Komori, S., 1999. Drag and lift forces on a rotating sphere in a linear shear flow. Journal of Fluid Mechanics 384, $183-206$.

Kurose, R., Misumi, R., Komori, S., 2001. Drag and lift forces acting on a spherical bubble in a linear shear air flow. International Journal of Multiphase Flow 27, 1247-1258.

Legendre, D., Magnaudet, J., 1997. A note on the lift force on a spherical bubble or drop in a low-Reynolds-number shear flow. Physics of Fluids 9, 3572-3574.

Legendre, D., Merle, A., Magnaudet, J., 2006. Wake of a spherical bubble or a solid sphere set fixed in a turbulent environment. Physics of Fluids 18, 048102.

Magnaudet, J., Takagi, S., Legendre, D., 2003. Drag, deformation and lateral migration of a buoyant drop moving near a wall. Journal of Fluid Mechanics 476, 115-157.

Maxworthy, T., 1965. Accurate measurements of sphere drag at low Reynolds numbers. Journal of Fluid Mechanics 23, 369-372.

Meliga, P., Chomaz, J.-M., Sipp, D., 2009. Global mode interaction and pattern selection in the wake of a disk: a weakly nonlinear expansion. Journal of Fluid Mechanics 633, 159-189.

Mittal, R., 1999. Planar symmetry in the unsteady wake of a sphere. AIAA Journal 37, 388-390.

Mittal, R., Iaccarino, G., 2005. Immersed boundary methods. Annual Review of Fluid Mechanics 37, $239-261$.

Nakamura, I., 1976. Steady wake behind a sphere. Physics of Fluids 19, 5-8.

Ormières, D., Provansal, M., 1999. Transition to turbulence in the wake of a sphere. Physical Review Letters 83, 80-83.

Pier, B., 2008. Local and global instabilities in the wake of a sphere. Journal of Fluid Mechanics 603, 39-61.

Rastello, M., Marié, J.-L., Grosjean, N., Lance, M., 2009. Drag and lift forces on interface-contaminated bubbles spinning in a rotating flow. Journal of Fluid Mechanics 624, 159-178.

Rastello, M., Marié, J.-L., Lance, M., 2011. Drag and lift forces on clean spherical and ellipsoidal bubbles in a solid-body rotating flow. Journal of Fluid Mechanics 682, 434-459.

Sakamoto, H., Haniu, H., 1995. The formation mechanism and shedding frequency of vortices from a sphere in uniform shear flow. Journal of Fluid Mechanics 287, 151-171.

Schouveiler, L., Provansal, M., 2002. Self-sustained oscillations in the wake of a sphere. Physics of Fluids 14, 3846-3854.

Sugioka, K.-I., Komori, S., 2007. Drag and lift forces acting on a spherical water droplet in homogenous linear shear air flow. Journal of Fluid Mechanics 570, 155-175.

Thompson, M. C., Leweke, T., Provansal, M., 2001. Kinematics and dynamics of sphere wake transition. Journal of Fluids and Structures $15,575-585$.

Tomboulides, A. G., Orszag, S. A., 2000. Numerical investigation of transitional and weak turbulent flow past a sphere. Journal of Fluid Mechanics 416, 45-73.

Veldhuis, C., Biesheuvel, A., van Wijngaarden, L., Lohse, D., 2005. Motion and wake structure of spherical particles. Nonlinearity $18, \mathrm{C} 1-\mathrm{C} 8$.

Zhang, N., Zheng, Z. C., 2007. An improved direct-forcing immersed-boundary method for finite difference applications. Journal of Computational Physics 221, 250-268. 